\title{
Fabrication of cellulose-based biopolymer optical fibers and their theoretical attenuation limit
}

Martin Reimer, Daniel Van Opdenbosch, and Cordt Zollfrank*

\section{AUTHOR INFORMATION}

Corresponding Author

Cordt Zollfrank - Technische Universität München, Chair for Biogenic Polymers, Campus Straubing for Biotechnology and Sustainability, Schulgasse 16, Straubing, 94315, Germany. E-mail: cordt.zollfrank@tum.de

\section{Authors}

Martin Reimer - Technische Universität München, Chair for Biogenic Polymers, Campus Straubing for Biotechnology and Sustainability, Schulgasse 16, Straubing, 94315, Germany. E-mail: martin.reimer@tum.de

Daniel Van Opdenbosch - Technische Universität München, Chair for Biogenic Polymers Campus Straubing for Biotechnology and Sustainability, Schulgasse 16, Straubing, 94315, Germany.

E-mail: daniel.van-opdenbosch@tum.de

\section{Supporting Information}



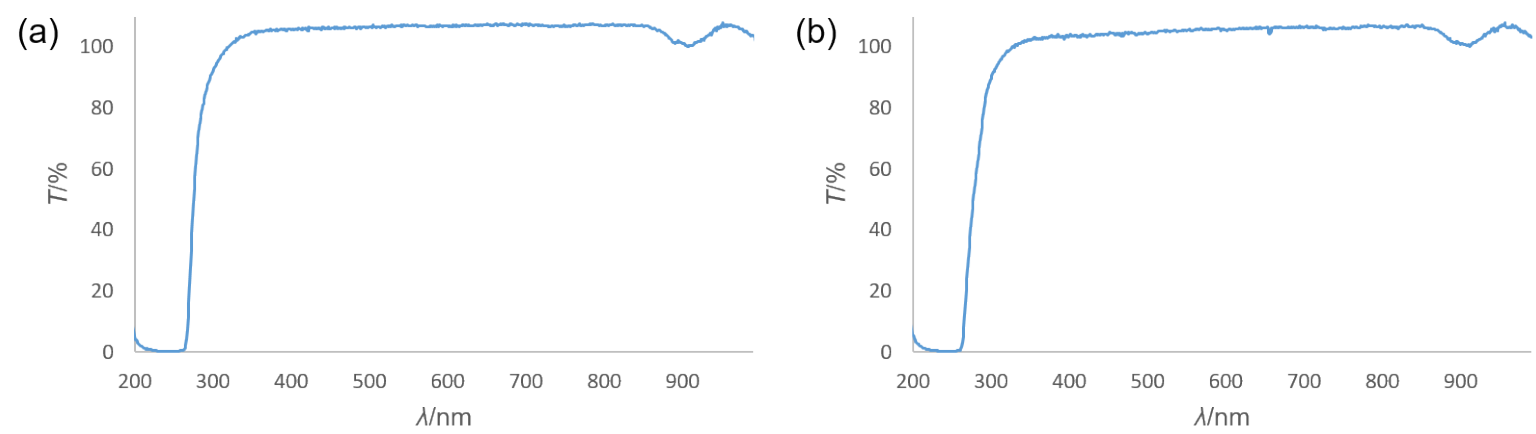

Supplementary Figure S1: Transmittance of (a) N,N-dimethylacetamide and (b) dissolved lithium chloride in

$N, N$-dimethylacetamide.

The transmission spectra of the solvents were measured using air-filled cuvettes as background. The spectra of the pure solvent $N, N$-dimethylacetamide as well as after the solution of lithium chloride have an identical course. They have a characteristic strong absorption band at about $250 \mathrm{~nm}$, as well as a small absorption band at $910 \mathrm{~nm}$. The difference in refractive index between glass and air is greater than between glass and solution. This results in greater reflection losses when measuring the background. These losses are reduced when measuring the sample filled with solution. As a result, transmission values of more than $100 \%$ can be achieved.
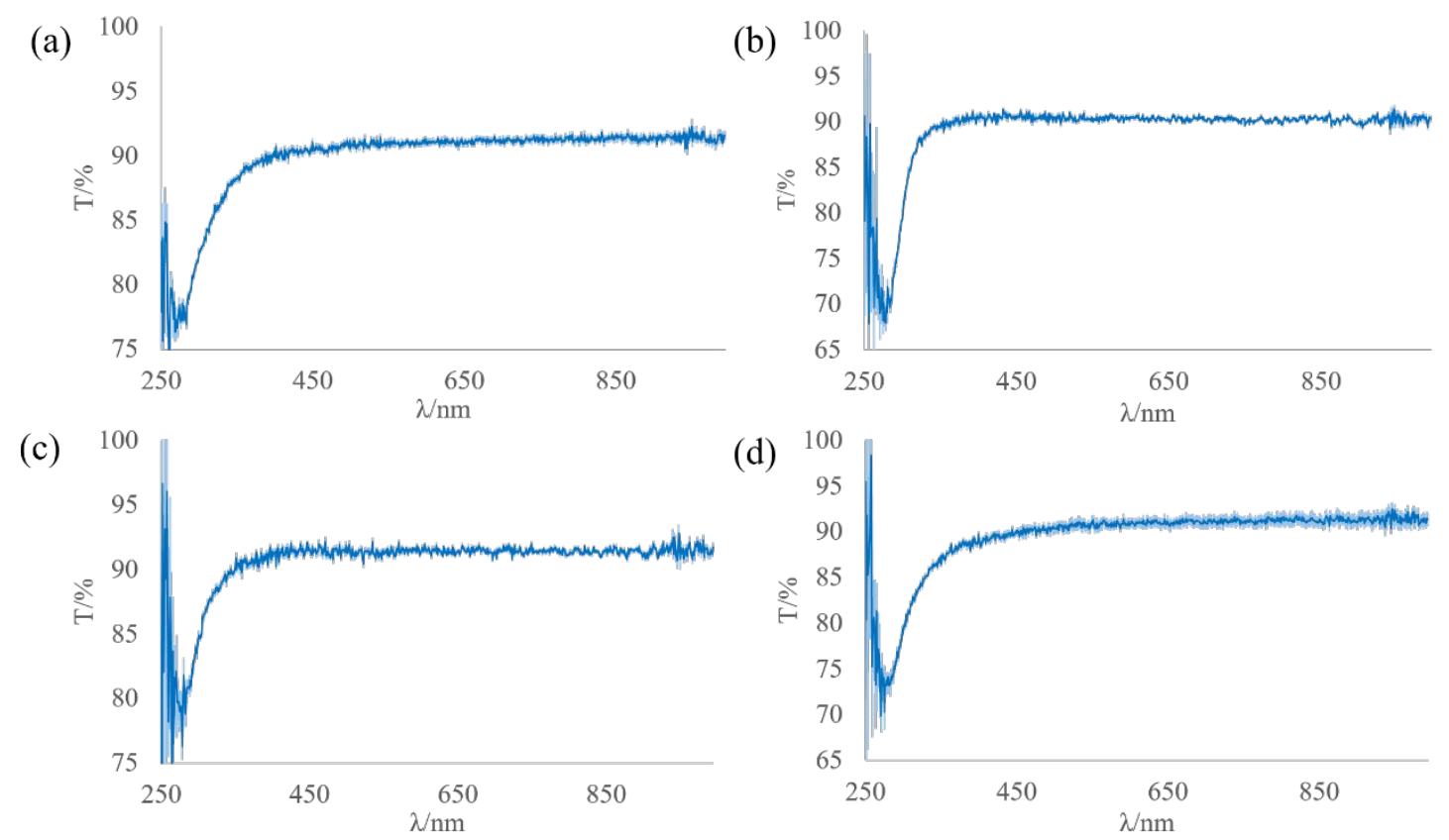

Supplementary Figure S2: Transmittance of (a) regenerated cellulose, (b) cellulose diacetate, (c) cellulose acetate propionate and (d) cellulose acetate butyrate films. 
The films made from regenerated cellulose, cellulose diacetate, cellulose acetate propionate and cellulose acetate butyrate have an absorption band at the wavelength of $275 \mathrm{~nm}$ and a transmission of about $90-92 \%$ in the visible range of the spectrum.

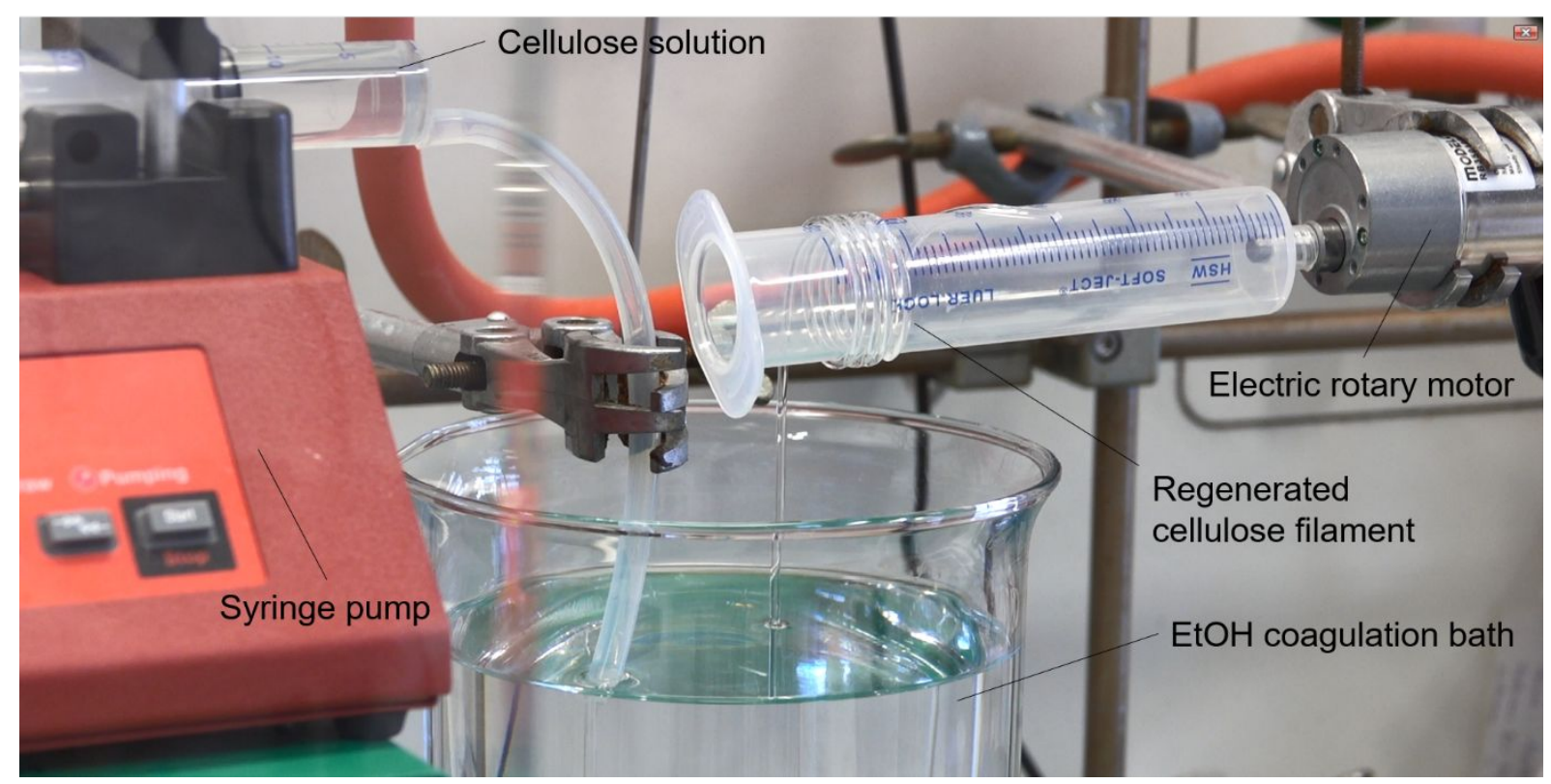

Supplementary Figure S3: Construction and implementation of the wet spinning process with syringe pump, colorless cellulose solution in DMAc/LiCL, EtOH coagulation bath, electric rotary motor and a continuous colorless and transparent regenerated cellulose filament.

The transparent solution in the syringe can be clearly seen, which after regeneration in EtOH, forms a transparent colorless and homogenous fiber being wound up in a dimensionally stable way. To ensure complete regeneration of the fibers, they are then stored for $1 \mathrm{~h}$ in $\mathrm{EtOH}$.

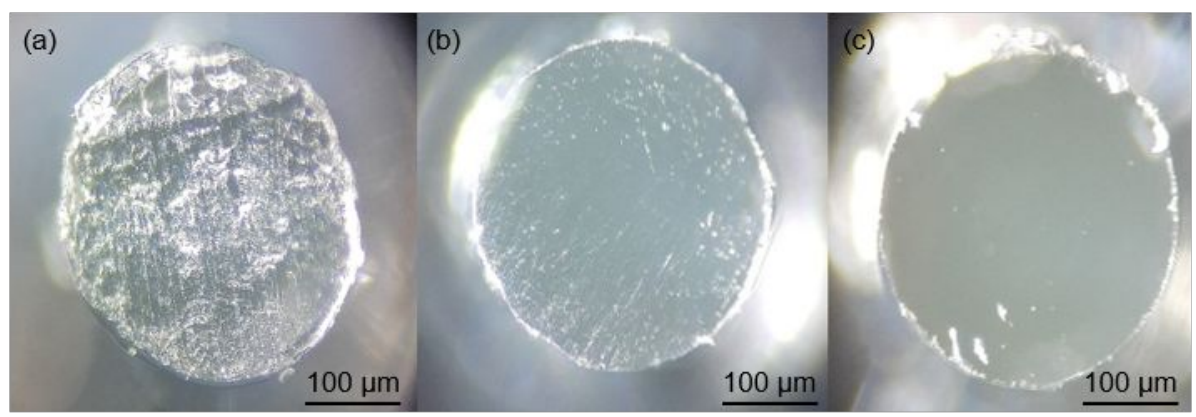

Supplementary Figure S4: Regenerated cellulose fiber facet (a) after cutting with a razor blade, (b) after polishing with an aluminum oxide lapping sheet (1 $\mu \mathrm{m}$ grit) and (c) after polishing with a calcined alumina lapping sheet $(0.3 \mu \mathrm{m}$ grit). 
The manufactured and air-dried regenerated cellulose fibers are cut with a razor blade (a). The fiber facet shows an irregular and rough surface. Then the fibers are polished with (b) an aluminium oxide lapping sheet ( $1 \mu \mathrm{m}$ grit) and (c) calcined alumina lapping sheet $(0.3 \mu \mathrm{m}$ grit). The polishing process produces a smooth and defect-free fiber facet. 